\title{
A SURROGATE MODELING APPROACH FOR ROCKING-FLEXURE INTERACTION
}

\author{
A PREPRINT \\ Andréia H. A. da Silva ${ }^{*}$ \\ Department of Civil, Environmental, \\ and Geomatic Engineering \\ ETH Zürich

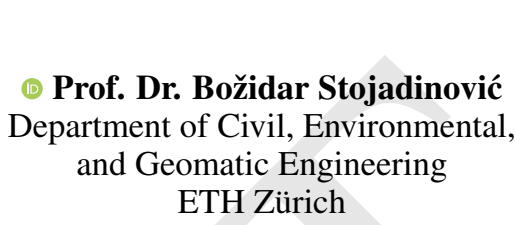

November $3^{\text {rd }}, 2021$

\section{Abstract}

Uplift of a structure is a seismic isolation technique: it works as a structural fuse limiting the maximum base moment (rocking foundation) or base shear (rocking podium) transferred to the foundation. To use uplifting in seismic design, the interaction between rocking and flexure must be understood well. Ideally, surrogate models to predict the seismic response of such systems should be also available to facilitate design. This paper presents the equations of motion of a building mounted on a rocking foundation and on a rocking podium in modal coordinates, then surrogate models are developed by applying component mode synthesis. Analytical equations for calculating the uplifted frequency and modal shapes are derived and used to compare how uplift affects both systems.In the following, it is shown that the overturning moment in both systems is dominated by the first mode, therefore a surrogate model with two degrees of freedom (2DOF surrogate), namely the rocking angle and the first mode coordinate, is considered adequate to represent the entire system. Finally, a hundred different buildings, with varying periods, aspect ratios and number of stories, are generated and the maximum dynamic response of the complete system is compared to the 2DOF surrogate ones, the coefficient of determination between all the responses is greater than 0.81 for all cases, thus the 2DOF surrogate is considered adequate to represent the response of the full model.

Keywords rocking, uplifting, rocking-flexure interaction

\section{Introduction}

Ordinary structures are usually attached to the ground, and sized to behave in flexure when a lateral load is applied. Nevertheless, after the 1960 Valdiva (Chile) earthquake, Housner [1] concluded that tall slender structures survived due to their possibility to uplift and rock, compared to severely damage, apparently more stable, fixed-base structures. When a structure is allowed to uplift during a ground motion, the moment transferred to the base is limited and there is no residual displacement, if there is no sliding. Thus, allowing a structure to uplift is a seismic design strategy. Inspired by the survival of ancient Greek temples over many centuries, Makris and Vassiliou [2] have shown the noticeable stability of an array of rocking columns when capped with a beam. Moreover, in Russia, rocking columns have been used to isolate buildings [3], while in New Zealand, rocking was used to seismically isolate bridges [4, 5] and a chimney [6].

Most of the literature describing the rocking behavior address rigid bodies, mostly because the interaction between rocking and flexure is complex. Nevertheless, there are several studies that address the rocking-flexure interaction. For a flexible structure allowed to uplift, Meek [7] investigated the dynamic response of a single mass structure rocking on a rigid foundation, Psycharis [8] investigated structures (single and multiple mass) rocking on a 2 spring and on a Winkler foundation, and Yim and Chopra [9, 10] developed a simplified method for analyzing those structures. More recently, Oliveto et al [11] developed the equations of motion of a flexible structure allowed to uplift subjected to large

${ }^{*}$ Corresponding author: Department of Civil, Environmental, and Geomatic Engineering, ETH Zürich. Stefano Francini Platz 5, 8093, Zurich, Switzerland. a.alvaresdasilva@ibk.baug.ethz.ch 
rotations, Vassiliou et al [12] investigated the behavior of a deformable cantilever with distributed and concentrated mass rocking on a rigid foundation, and Acikgoz and DeJong [13] investigated the behavior of a multiple degree of freedom rocking on a rigid foundation.

Rocking-flexure interaction can also be observed in the rocking podium systems. Bachmann et al [14] developed the equations of motion for a single mass mounted on a rocking podium and investigated its stability when subjected to pulse and natural ground motions. Later, Bantilas et al [15] analyzed the behavior of a multiple-degrees-of-freedom (MDOF) system mounted on a rocking podium, to understand how higher modes affects the behavior of the system. And, more recently, Bantilas et al [16] developed fragility curves for rocking podium systems, using synthetic pulses and analyzing the performance of multiple intensity measures for this system.

In most of the aforementioned studies, the flexural structure is often represented by a single-degree-of-freedom (SDOF) system for simplicity. This approach reduces the number of variables of the problem and makes it easier for deriving conclusions that can be extended to MDOF systems. Modal dynamic response decomposition was used in several previous studies $[8,9 ;, 12])$ to account of the flexural properties of the system. The reduction of a part of the dynamic response coordinates using modal decomposition belong to a class of component modes synthesis techniques [17; 18], which is widely applied in the aeronautic field.

Herein, component modes synthesis is applied to produce a surrogate model that accounts rocking and flexure behaviors, and to represent the seismic response of a structure on a rocking foundation and/or rocking podium. A successful surrogate model has to be simple enough, i.e., described by few parameters, and accurate enough to be used for seismic design of rocking systems. Therefore, a two degrees of freedom (2DOF) surrogate is chosen to represent the full system, the DOFs are: the rocking angle and the first mode of the structure coordinate. Different methods are used to assess if the surrogate is adequate. First, analytical expressions for calculating the modal properties of the uplifted system are developed and used to assess how uplift influences higher modes. Then, the influence of higher modes on uplift is also analyzed, by computing the contribution of each mode to the dynamic responses (i.e. displacement, rotation and base shear). Finally, the accuracy of the surrogate model prediction of maximum dynamic responses is assessed by computing the coefficient of determination relative to the full model maximum dynamic responses.

\section{Systems Analyzed}

Two different systems are analyzed herein: a building on a rocking foundation and a building on a rocking podium. Both systems present a rocking-flexure interaction problem and are base isolated as the base shear and moments are limited by the rocking motion. In this section, it is shown that the equations of motion of both systems are mathematically very similar, but the parameters governing the rocking motion are different, that is: on a rocking foundation the height and aspect ratio of the entire building governs the rocking motion, while in the rocking podium it is the height and size of the first-floor columns. Moreover, the isolation behaviors are different: the rocking foundation system rotates around the corners, while the rocking podium system works similarly to a sliding base, i.e. it controls the motion transferred horizontally to the base of the superstructure. The equations of motion of the full systems and of the surrogate models are developed in this section, they are of central importance for the production of earthquake spectra that enable the use of those systems in seismic design.

\subsection{Multiple degrees of freedom system on a rocking foundation}

The equations of motion of a MDOF system on a rocking foundation (Figure 1 ) are presented herein following the work of Psycharis [8], Yim and Chopra [9] and Acikgoz and DeJong [13]. The MDOF system has $N+1$ lumped masses and a rigid foundation of width $2 B$. The distance between the masses $\left(m_{i}\right)$ and the base mass $\left(m_{b}\right)$ is given by the vector of heights $\left(\boldsymbol{\iota}_{\boldsymbol{h}}=\left[h_{1} h_{2} \ldots h_{N}\right]^{T}\right)$. The fixed MDOF system is represented by a mass matrix $\left(\boldsymbol{m}_{\boldsymbol{s}}\right)$ and a elastic stiffness matrix $\left(\boldsymbol{k}_{\boldsymbol{s}}\right)$, which is condensed to consider only the horizontal degrees of freedom. The fixed MDOF system damping is modeled using superposition of modal damping matrices [19], that is all modes have the same damping ratio $(\zeta)$ summing up to a damping matrix $c_{s}$. The system is excited by a horizontal ground motion $\left(\ddot{u}_{g}\right)$ and moves in-plane. The resulting motions of the masses are described by the base rotation $(\theta)$ and the horizontal translation $\left(\boldsymbol{u}_{s}\right)$ of the story masses relative to the base mass, assuming small rotations and that the system base does not slide.

The equation of motion of the system before uplift is:

$$
\boldsymbol{m}_{s} \ddot{u}_{s}+\boldsymbol{c}_{s} \dot{\boldsymbol{u}}_{s}+\boldsymbol{k}_{s} \boldsymbol{u}_{s}=-\boldsymbol{m}_{s} \iota \ddot{u}_{g}
$$

in which $\iota$ is a column vector of ones. The uplift condition is: the absolute value of overturning moment $\left(M_{o}\right)$ must be greater than the resisting moment $\left(M_{r}\right)$. Thus:

$$
\left|M_{o}\right|=\left|\boldsymbol{\iota}_{\boldsymbol{h}}^{\boldsymbol{T}} \boldsymbol{m}_{\boldsymbol{s}}\left(\ddot{\boldsymbol{u}}_{\boldsymbol{s}}+\boldsymbol{\iota} \ddot{u}_{g}\right)\right|>\left(\boldsymbol{\iota}^{\boldsymbol{T}} \boldsymbol{m}_{\boldsymbol{s}} \iota+m_{b}\right) g B=M_{r}
$$




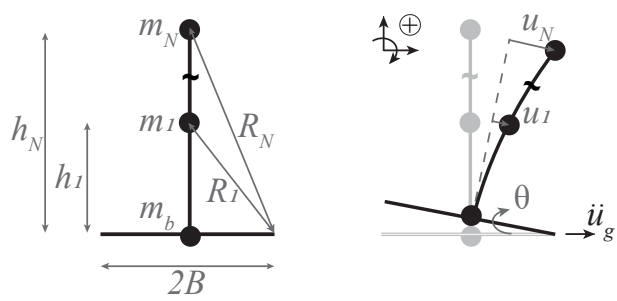

Figure 1: Flexible multiple degrees of freedom system with a rocking foundation.

if the overturning moment is positive, the structure rotates around the left pivot point $(\theta<0)$; respectively, if $M_{O}$ is negative, the structure rotates around the right pivot point $(\theta>0)$. After uplift, the linearized equation of motion, considering small displacements, is:

$$
\left[\begin{array}{cc}
I_{\theta} & \left(\boldsymbol{m}_{\boldsymbol{s}} \boldsymbol{\iota}_{\boldsymbol{h}}\right)^{T} \\
\boldsymbol{m}_{\boldsymbol{s}} \boldsymbol{\iota}_{\boldsymbol{h}} & \boldsymbol{m}_{\boldsymbol{s}}
\end{array}\right]\left[\begin{array}{c}
\ddot{\theta} \\
\ddot{\boldsymbol{u}}_{\boldsymbol{s}}
\end{array}\right]+\left[\begin{array}{cc}
0 & \mathbf{0} \\
\mathbf{0} & \boldsymbol{c}_{\boldsymbol{s}}
\end{array}\right]\left[\begin{array}{c}
\ddot{\theta} \\
\dot{\boldsymbol{u}}_{\boldsymbol{s}}
\end{array}\right]+\left[\begin{array}{cc}
-L_{0}^{r} g & \mathbf{0} \\
\mathbf{0} & \boldsymbol{k}_{\boldsymbol{s}}
\end{array}\right]\left[\begin{array}{c}
\theta \\
\boldsymbol{u}_{\boldsymbol{s}}
\end{array}\right] \pm\left[\begin{array}{c}
m_{t} g B \\
\mathbf{0}
\end{array}\right]=-\left[\begin{array}{c}
L_{0}^{r} \\
\boldsymbol{m}_{\boldsymbol{s}} \boldsymbol{\iota}
\end{array}\right] \ddot{u}_{g}
$$

in which $I_{\theta}$ is the rotational inertia: $I_{\theta}=\boldsymbol{\iota}_{\boldsymbol{R}}^{\boldsymbol{T}} \boldsymbol{m}_{\boldsymbol{s}} \iota_{\boldsymbol{R}}+m_{b} B^{2}$, and the distance of masses to the pivot point is given by a vector of radii $\iota_{\boldsymbol{R}}=\left[\begin{array}{lll}R_{1} & R_{2} \ldots\end{array}\right]^{T}$. The sum of story mass and height products is $L_{0}^{r}=\boldsymbol{\iota}^{\boldsymbol{T}} \boldsymbol{m}_{\boldsymbol{s}} \boldsymbol{\iota}_{\boldsymbol{h}}$, and the total mass of the system is $m_{t}=\boldsymbol{\iota}^{\boldsymbol{T}} \boldsymbol{m}_{\boldsymbol{s}} \iota+m_{b}$. Lastly, the upper, positive, sign is for rotation around the right pivot, while the lower, negative, sign is for rotation around the left pivot.

After uplift, the structure can overturn or impact the ground. The classical impact model is based on the conservation of angular momentum, and it assumes that the impact is instantaneous and the impact forces are concentrated at the impact point, located on the base edge (Housner [1] approach). Even though this model is widely applied for rocking blocks, for flexible bodies on rigid foundations different models are available (e.g., Meek [7] and Acikgoz and DeJong [20]). A review of different methods and analysis of their coherence is available in Vassiliou et al [12] who also validated the impact models against experimental results in [21]. Following their work, herein the impact model named Vertical Velocity Energy Loss (VVEL) is adopted, which is the same adopted in [7, 9, 22]. In this model, the collision between foundation and ground is perfectly inelastic without bouncing, thus completely dissipating the vertical velocity, i.e., $\dot{\theta}$ is equal to zero after impact and the structure sticks to the ground. Meanwhile, the horizontal kinetic energy of the masses is conserved. Thus, the horizontal kinetic energy before $\left(E_{1, h k}\right)$ and total kinetic energy after impact $\left(E_{2, k}\right)$ must be equal. The horizontal kinetic energy before impact is:

$$
E_{1, h k}=\sum_{i=1}^{N} \frac{1}{2} m_{i}\left(h_{i} \dot{\theta}_{i, 1}+\dot{u}_{1}\right)^{2}
$$

in which the subscript 1 denotes before impact and 2 after impact. The total kinetic energy after impact is:

$$
E_{2, k}=\sum_{i=1}^{N} \frac{1}{2} m_{i} \dot{u}_{i, 2}^{2}
$$

by equating $E_{1, h k}$ and $E_{2, k}$ only one equation for $N$ unknowns $\left(\dot{u}_{i, 2}\right)$ is available. Therefore, it is assumed that each degree of freedom will have its horizontal kinetic energy conserved:

$$
\dot{\boldsymbol{u}}_{2}=\dot{\boldsymbol{u}}_{\mathbf{1}}+\iota_{\boldsymbol{h}} \dot{\theta}_{1}
$$

\subsection{Surrogate model of the rocking foundation system}

A coordinate transformation of the equation of motion (Equation 3) enables the construction of a surrogate model. The technique used is named component mode synthesis [17; 18]. For the rocking-flexure interaction problem, it was first applied by Psycharis [22] followed by Yim and Chopra [9]. The main steps followed are: (i) from a problem described in global coordinates, divide the structure between internal DOFs and boundary DOFs, (ii) change the coordinates such as the displacements of the internal DOFs are relative to the boundary ones, (iii) assuming the boundary DOFs as fixed, do a modal analysis of the substructure containing the internal DOFs, (iv) transform the internal DOFs to modal coordinates, by applying the mode shapes, (v) suppress the higher modes as long as they are not important for the global response.

Equation 3 already presents the problem in a way that the internal DOFs displacements $\left(\boldsymbol{u}_{\boldsymbol{s}}\right)$ are relative to the boundary DOF $(\theta)$. Thus, the next step is performing a modal analysis of the substructure: $\boldsymbol{k}_{\boldsymbol{s}} \boldsymbol{\Phi}=\boldsymbol{m}_{\boldsymbol{s}} \boldsymbol{\Phi} \boldsymbol{\Omega}^{2}$. Then, 
pre-multiplying the $2^{\text {nd }}$ line to the $N+1^{\text {th }}$ line of Equation 3 by $\left(\boldsymbol{\Phi}^{T} \boldsymbol{m}_{\boldsymbol{s}} \boldsymbol{\Phi}\right)^{-1} \boldsymbol{\Phi}^{T}$ and applying the modal coordinate change $\boldsymbol{u}_{s}=\boldsymbol{\Phi} \boldsymbol{q}$, gives:

$$
\left[\begin{array}{cc}
I_{\theta} & \boldsymbol{L}^{\boldsymbol{\theta}^{T}} \\
\boldsymbol{\Gamma}_{\boldsymbol{\theta}} & \mathbf{1}
\end{array}\right]\left[\begin{array}{l}
\ddot{\theta} \\
\ddot{\boldsymbol{q}}
\end{array}\right]+\left[\begin{array}{cc}
0 & \mathbf{0} \\
\mathbf{0} & \operatorname{diag}\left(2 \zeta \omega_{n}\right)
\end{array}\right]\left[\begin{array}{l}
\dot{\theta} \\
\dot{\boldsymbol{q}}
\end{array}\right]+\left[\begin{array}{cc}
-L_{0}^{r} g & \mathbf{0} \\
\mathbf{0} & \operatorname{diag}\left(\omega_{n}^{2}\right)
\end{array}\right]\left[\begin{array}{c}
\theta \\
\boldsymbol{q}
\end{array}\right] \pm\left[\begin{array}{c}
m_{t} g B \\
\mathbf{0}
\end{array}\right]=-\left[\begin{array}{c}
L_{0}^{r} \\
\boldsymbol{\Gamma}
\end{array}\right] \ddot{u}_{g}
$$

in which $\omega_{n}$ is the natural frequencies of the fixed-base system, thus $\left(\boldsymbol{\Phi}^{\boldsymbol{T}} \boldsymbol{m}_{\boldsymbol{s}} \boldsymbol{\Phi}\right)^{-1}\left(\boldsymbol{\Phi}^{\boldsymbol{T}} \boldsymbol{k}_{s} \boldsymbol{\Phi}\right)=\operatorname{diag}\left(\omega_{n}^{2}\right)$, as $\boldsymbol{c}_{\boldsymbol{s}}$ is built by superposition of modal damping matrices: $\left(\boldsymbol{\Phi}^{\boldsymbol{T}} \boldsymbol{m}_{\boldsymbol{s}} \boldsymbol{\Phi}\right)^{-\mathbf{1}}\left(\boldsymbol{\Phi}^{\boldsymbol{T}} \boldsymbol{c}_{\boldsymbol{s}} \boldsymbol{\Phi}\right)=\operatorname{diag}\left(2 \zeta \omega_{n}\right)$. Then, the modal parameters are defined:

$$
\begin{gathered}
L^{h}=\Phi^{T} m_{s} \iota \\
L^{\theta}=\Phi^{T} m_{s} \iota_{h} \\
\Gamma=\frac{\Phi^{T} m_{s} \iota}{\Phi^{T} m_{s} \Phi} \\
\Gamma_{\theta}=\frac{\Phi^{T} m_{s} \iota_{h}}{\Phi^{T} m_{s} \Phi}
\end{gathered}
$$

Equation 7 can be further simplified by applying the coordinate change: $q_{i}=\Gamma_{i} D_{i}$ :

$$
\left[\begin{array}{cc}
I_{\theta} & \left(\operatorname{diag}\left(\boldsymbol{m}^{*}\right) \boldsymbol{h}^{*}\right)^{T} \\
\boldsymbol{h}^{*} & \mathbf{1}
\end{array}\right]\left[\begin{array}{c}
\ddot{\theta} \\
\ddot{\boldsymbol{D}}
\end{array}\right]+\left[\begin{array}{cc}
0 & \mathbf{0} \\
\mathbf{0} & \operatorname{diag}\left(2 \zeta \omega_{n}\right)
\end{array}\right]\left[\begin{array}{c}
\dot{\theta} \\
\dot{\boldsymbol{D}}
\end{array}\right]+\left[\begin{array}{cc}
-L_{0}^{r} g & \mathbf{0} \\
\mathbf{0} & \operatorname{diag}\left(\omega_{n}^{2}\right)
\end{array}\right]\left[\begin{array}{c}
\theta \\
\boldsymbol{D}
\end{array}\right] \pm\left[\begin{array}{c}
m_{t} g B \\
\mathbf{0}
\end{array}\right]=-\left[\begin{array}{c}
L_{0}^{r} \\
\mathbf{1}
\end{array}\right] \ddot{u}_{g}
$$

in which $\boldsymbol{m}^{*}$ and $\boldsymbol{h}^{*}$ are column vectors with the following elements:

$$
\begin{gathered}
m_{i}^{*}=\frac{\left(L_{i}^{h}\right)^{2}}{\phi_{i}^{T} \boldsymbol{m}_{\boldsymbol{s}} \phi_{i}} \\
h_{i}^{*}=\frac{L_{i}^{\theta}}{L_{i}^{h}}
\end{gathered}
$$

in which $\phi_{i}$ is the $i^{\text {th }}$ column vector of $\boldsymbol{\Phi}$.

Lastly, the rocking initiation condition (Equation 2) is calculated in modal coordinates by:

$$
\left|\left(\boldsymbol{L}^{\boldsymbol{\theta}}\right)^{T} \ddot{\boldsymbol{q}}+L_{0}^{r} \ddot{u}_{g}\right|=\left|\left(\operatorname{diag}\left(\boldsymbol{m}^{*}\right) \boldsymbol{h}^{*}\right)^{T} \ddot{\boldsymbol{D}}+L_{0}^{r} \ddot{u}_{g}\right|>m_{t} g B
$$

and the post-impact velocities (Equation 6):

$$
\dot{\boldsymbol{q}}_{2}=\dot{\boldsymbol{q}}_{\mathbf{1}}+\boldsymbol{\Phi}^{-1} \iota_{\boldsymbol{h}} \dot{\theta}_{1}
$$

note that the multiplier of $\dot{\theta}_{1}\left(\boldsymbol{\Phi}^{-1} \iota_{\boldsymbol{h}}\right)$ is possible to be computed using only the eigenvectors of the modes of interest:

$$
\boldsymbol{\Phi}^{-\boldsymbol{1}} \iota_{\boldsymbol{h}}=\frac{\boldsymbol{\Phi}^{\boldsymbol{T}} \boldsymbol{l}_{\boldsymbol{h}}}{\boldsymbol{\Phi}^{\boldsymbol{T}} \boldsymbol{\Phi}}=\left[\begin{array}{c}
\phi_{1}^{T} \iota_{\boldsymbol{h}} /\left(\phi_{1}^{T} \phi_{1}\right) \\
\vdots \\
\phi_{n}^{T} \iota_{\boldsymbol{h}} /\left(\phi_{n}^{T} \phi_{n}\right)
\end{array}\right]
$$

therefore:

$$
\dot{D}_{i, 2}=\dot{D}_{i, 1}+h_{i}^{*} \dot{\theta}_{1}
$$

Equation 12 is the same as the one derived in Yim and Chopra [9] and follows Chopra's notation [19]. And, as they observed, if only the first mode is considered and all higher modes are suppressed, the remaining two equations are almost the same as a single mass system on a rocking foundation with mass $m_{1}^{*}$ and height $h_{1}^{*}$, given some base properties changes (adding mass and rotational inertia to the base). 


\subsection{Multiple degrees of freedom system on a rocking podium}

The equations of motion of a MDOF system on a rocking podium (Figure 2) are presented herein following the work of Makris and Vassiliou [2], Bachmann et al [14] and Bantilas et al [15]. The superstructure has $N$ lumped masses, it is flexible and has an elastic stiffness matrix $\left(\boldsymbol{k}_{\boldsymbol{s}}\right)$, which is condensed to consider only the horizontal degrees of freedom, a diagonal mass matrix $\left(\boldsymbol{m}_{\boldsymbol{s}}\right)$ and a damping matrix $\left(\boldsymbol{c}_{\boldsymbol{s}}\right)$ constructed by superposition of modal damping matrices [19],in which $\zeta$ is the damping ratio of each mode. The rocking podium consists of a rigid cap beam of total mass $m_{b}$ and $N_{c}$ rigid rocking columns, each of total mass $m_{c}$. The mass density of the cap beam and the rocking columns is uniform. Each rocking column is rectangular, with geometry defined by the base width $2 B$ and the height $2 H$ (thus slenderness $\alpha=\tan ^{-1}(B / H)$ and semi-diagonal $\left.R=\sqrt{B^{2}+H^{2}}\right)$ The system is excited by a horizontal ground motion $\left(\ddot{u}_{g}\right)$. The system moves in-plane, but the rocking columns do not slide. The resulting displacement of the masses are assumed to be small (i.e., $P-\Delta$ effects are not considered).
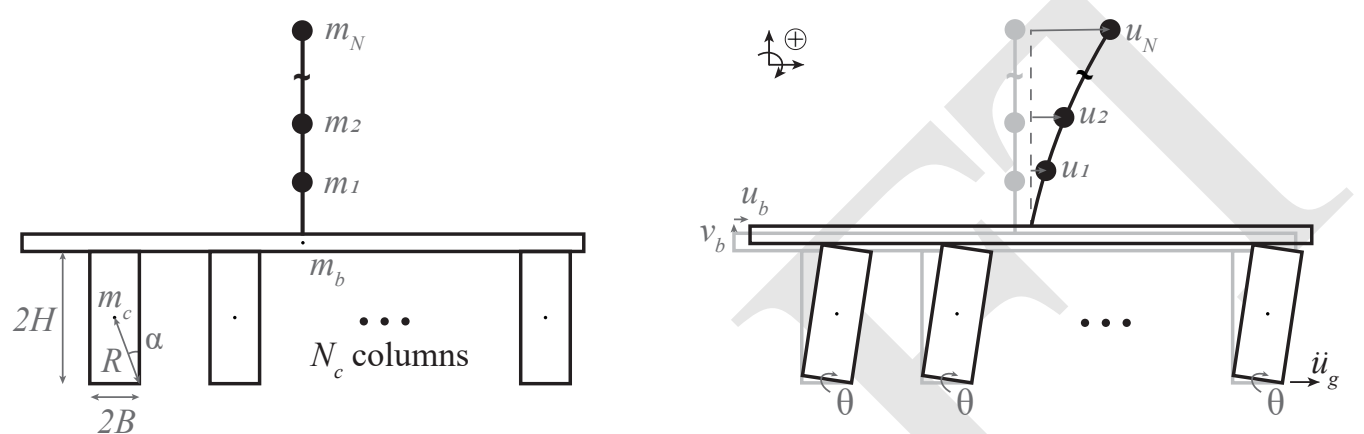

Figure 2: Flexible multiple degrees of freedom system on top of a rocking podium.

Before columns uplift, the system equation of motion is exactly Equation 1 The condition for uplifting is: the absolute value of the overturning moment $\left(\left|M_{o}\right|\right)$ must be greater than the resisting moment $\left(M_{r}\right)$ :

$$
\left|M_{o}\right|=\left|M_{\theta} H \ddot{u}_{g}+2 H \iota^{\boldsymbol{T}} \boldsymbol{m}_{\boldsymbol{s}} \ddot{\boldsymbol{u}}_{\boldsymbol{s}}\right|>M_{\theta} g B=M_{r}
$$

in which $M_{\theta}=N_{c} m_{c}+2 m_{b}+2\left(\iota^{\boldsymbol{T}} \boldsymbol{m}_{\boldsymbol{s}} \boldsymbol{\iota}\right)$. If the overturning moment is positive, the columns rotate around the left pivot (resp. if $M_{o}$ is negative, the columns rotate around the right pivot, i.e., $\theta>0$ ). After uplift, linearizing the equations in [15] and denoting $\boldsymbol{u}_{\boldsymbol{s}}$ the displacement of the masses relative to the cap beam, the equation of motion is:

$$
\left[\begin{array}{cc}
I_{\theta, p} & 2 H\left(\boldsymbol{\iota}^{\boldsymbol{T}} \boldsymbol{m}_{\boldsymbol{s}}\right) \\
2 H\left(\boldsymbol{m}_{\boldsymbol{s}} \boldsymbol{\iota}\right) & \boldsymbol{m}_{\boldsymbol{s}}
\end{array}\right]\left[\begin{array}{c}
\ddot{\theta} \\
\ddot{\boldsymbol{u}}_{\boldsymbol{s}}
\end{array}\right]+\left[\begin{array}{cc}
0 & \mathbf{0} \\
\mathbf{0} & \boldsymbol{c}_{\boldsymbol{s}}
\end{array}\right]\left[\begin{array}{c}
\dot{\theta} \\
\dot{\boldsymbol{u}}_{\boldsymbol{s}}
\end{array}\right]+\left[\begin{array}{cc}
-M_{\theta} g H & \mathbf{0} \\
\mathbf{0} & \boldsymbol{k}_{\boldsymbol{s}}
\end{array}\right]\left[\begin{array}{c}
\theta \\
\boldsymbol{u}_{\boldsymbol{s}}
\end{array}\right] \pm\left[\begin{array}{c}
M_{\theta} g B \\
\mathbf{0}
\end{array}\right]=-\left[\begin{array}{c}
M_{\theta} H \\
\boldsymbol{m}_{\boldsymbol{s}} \boldsymbol{\iota}
\end{array}\right] \ddot{u}_{g}
$$

in which $I_{\theta, p}=(4 / 3) N_{c} m_{c} R^{2}+4 m_{b} R^{2}+4\left(\boldsymbol{\iota}^{\boldsymbol{T}} \boldsymbol{m}_{\boldsymbol{s}} \boldsymbol{\iota}\right) R^{2}$, and the upper, positive, sign is for rotation around the right pivot and the lower, negative, sign is for rotation around the left pivot.

After uplift, the columns can overturn or impact the ground. In the podium case, the columns are considered to be acting as rigid blocks, thus the classical impact model proposed by Housner [1] is adopted, following the work of Bachmann et al [14]. They assumed that (i) the impact is instantaneous; (ii) the impact forces are equal in all columns and concentrated at the pivot points; (iii) the displacement of the superstructure is small compared to its total size; (iv) the horizontal velocity of the superstructure masses relative to the cap beam does not change during impact $\left(\dot{\boldsymbol{u}}_{s, 2}=\dot{\boldsymbol{u}}_{\boldsymbol{s}, 1}\right)$. This leads to the following coefficient of restitution:

$$
r=\frac{\dot{\theta}_{2}}{\dot{\theta}_{1}}=\left[1-2 \sin ^{2}(\alpha) \frac{N m_{c}+4 m_{b}+4\left(\boldsymbol{\iota}^{\boldsymbol{T}} \boldsymbol{m}_{\boldsymbol{s}} \boldsymbol{\iota}\right)}{(4 / 3) N m_{c}+4 m_{b}+4\left(\boldsymbol{\iota}^{\boldsymbol{T}} \boldsymbol{m}_{\boldsymbol{s}} \boldsymbol{\iota}\right)}\right]
$$

thus, the angular velocity of the blocks after impact is given by $\dot{\theta}_{2}=r \dot{\theta}_{1}$.

\subsection{Surrogate model of the rocking podium system}

The component mode synthesis technique is applied to Equation 20 The boundary DOF is the displacement of the cap beam, which is completely described by the rotation of the rocking columns $(\theta)$. As the superstructure displacements are already relative to the cap beam displacement, the necessary step is to change the coordinates of the superstructure's 
DOFs by modal coordinates. Then, pre-multiplying the $2^{\text {nd }}$ line to the $N+l^{\text {th }}$ line of Equation 20 by $\left(\boldsymbol{\Phi}^{\boldsymbol{T}} \boldsymbol{m}_{\boldsymbol{s}} \boldsymbol{\Phi}\right)^{-1} \boldsymbol{\Phi}^{T}$ and applying the modal coordinate change $u_{s}=\Phi q$ (in which $k_{s} \Phi=m_{s} \Phi \Omega^{2}$ ), results in:

$$
\left[\begin{array}{cc}
I_{\theta, p} & 2 H\left(\boldsymbol{L}^{\boldsymbol{h}}\right)^{T} \\
2 H \boldsymbol{\Gamma} & \mathbf{1}
\end{array}\right]\left[\begin{array}{l}
\ddot{\theta} \\
\ddot{\boldsymbol{q}}
\end{array}\right]+\left[\begin{array}{cc}
0 & \mathbf{0} \\
\mathbf{0} & \operatorname{diag}\left(2 \zeta \omega_{n}\right)
\end{array}\right]\left[\begin{array}{l}
\dot{\theta} \\
\dot{\boldsymbol{q}}
\end{array}\right]+\left[\begin{array}{cc}
-M_{\theta} g H & \mathbf{0} \\
\mathbf{0} & \operatorname{diag}\left(\omega_{n}^{2}\right)
\end{array}\right]\left[\begin{array}{l}
\theta \\
\boldsymbol{q}
\end{array}\right] \pm\left[\begin{array}{c}
M_{\theta} g B \\
\mathbf{0}
\end{array}\right]=-\left[\begin{array}{c}
M_{\theta} H \\
\boldsymbol{\Gamma}
\end{array}\right] \ddot{u}_{g}
$$

Equation 22 can be further simplified if the coordinate change $q_{i}=\Gamma_{i} D_{i}$ is applied:

$$
\left[\begin{array}{cc}
I_{\theta, p} & 2 H\left(\boldsymbol{m}^{*}\right)^{T} \\
2 H & \mathbf{1}
\end{array}\right]\left[\begin{array}{c}
\ddot{\theta} \\
\ddot{\boldsymbol{D}}
\end{array}\right]+\left[\begin{array}{cc}
0 & \mathbf{0} \\
\mathbf{0} & \operatorname{diag}\left(2 \zeta \omega_{n}\right)
\end{array}\right]\left[\begin{array}{c}
\dot{\theta} \\
\dot{\boldsymbol{D}}
\end{array}\right]+\left[\begin{array}{cc}
-M_{\theta} g H & \mathbf{0} \\
\mathbf{0} & \operatorname{diag}\left(\omega_{n}^{2}\right)
\end{array}\right]\left[\begin{array}{c}
\theta \\
\boldsymbol{D}
\end{array}\right] \pm\left[\begin{array}{c}
M_{\theta} g B \\
\mathbf{0}
\end{array}\right]=-\left[\begin{array}{c}
M_{\theta} H \\
\mathbf{1}
\end{array}\right] \ddot{u}_{g}
$$

Lastly the condition for rocking initiation in modal coordinates is:

$$
\left|M_{\theta} H \ddot{u}_{g}+2 H\left(\boldsymbol{L}^{\boldsymbol{h}}\right)^{T} \ddot{\boldsymbol{q}}\right|=\left|M_{\theta} H \ddot{u}_{g}+2 H\left(\boldsymbol{m}^{*}\right)^{T} \ddot{\boldsymbol{D}}\right| \geq M_{\theta} g B
$$

It is clear the similarity between Equations 12 and 23 Also, if only the first mode is considered and all higher modes are suppressed, Equation 23 is very similar to a single mass on a rocking podium. The main difference is the value of $M_{\theta}$ and $I_{\theta, p}$ : for the modal case (Equation 23, it is calculated with the total mass of the superstructure $\left(\boldsymbol{\iota}^{\boldsymbol{T}} \boldsymbol{m}_{\boldsymbol{s}} \boldsymbol{\iota}\right)$ even if the single mass is $m_{1}^{*}$ and, for a single mass on a podium, $M_{\theta}$ and $I_{\theta, p}$ are calculated with the single mass considered. Yet, it is possible to adjust the mass of the cap beam to match the values of $M_{\theta}$ and $I_{\theta, p}$ calculated with the entire mass, thus: $m_{b, n e w}=m_{b}+\left(\iota^{\boldsymbol{T}} \boldsymbol{m}_{\boldsymbol{s}} \boldsymbol{\iota}\right)-m_{1}^{*}$.

\subsection{Computation of vibration properties}

For both the rocking foundation and rocking podium cases, it is possible to do modal analysis of the uplifted MDOF system (e.g., as Acikgoz and DeJong [13]). However, for simplicity, the non-homogeneous damping matrix is replaced by a classical one to perform a classical eigenvalue analysis. As the damping ratio of the fixed MDOF system is expected to be low (i.e., $\zeta \leq 5 \%$ ) the incurred error is expected to be acceptably small. Thus, the mass and stiffness matrices in Equations 12 and 23 are used to calculate the uplifted natural frequencies $\left(\omega_{n}^{*}\right)$ and the mode shapes $\left(\phi_{n}^{*}\right)$.

For the rocking foundation case:

$$
\left(\left[\begin{array}{cc}
-L_{0}^{r} g / I_{\theta} & 0 \\
0 & \operatorname{diag}\left(\omega_{n}^{2}\right)
\end{array}\right]-\left[\begin{array}{cc}
1 & \boldsymbol{L}^{\boldsymbol{\theta}^{T}} / I_{\theta} \\
\boldsymbol{\Gamma}_{\boldsymbol{\theta}} & \mathbf{1}
\end{array}\right] \omega_{n}^{* 2}\right) \phi_{n}^{*}=\mathbf{0}
$$

after naming the eigenvectors' components: $\phi_{n}^{*}=\left[\phi_{0, n}^{*} \phi_{1, n}^{*} \cdots \phi_{N, n}^{*}\right]^{T}$, the second line of Equation 25 becomes:

$$
\phi_{i, n}^{*}=\phi_{0, n}^{*} \frac{\Gamma_{\theta, i} \omega_{n}^{* 2}}{\omega_{i}^{2}-\omega_{n}^{* 2}} \quad \text { for } \mathrm{i}=1 \text { until } N \text { and } \mathrm{n}=0 \text { until } N
$$

Substituting in the first line of Equation 25, and naming $p^{2}=-L_{0}^{r} g / I_{\theta}$ the rocking frequency, it follows that:

$$
\left(p^{2}-\omega_{n}^{* 2}\right) \phi_{0, n}^{*}+\sum_{i=1}^{N}\left(\frac{-\left(L_{i}^{\theta} / I_{\theta}\right) \Gamma_{\theta, i} \omega_{n}^{* 4}}{\omega_{i}^{2}-\omega_{n}^{* 2}}\right) \phi_{0, n}^{*}=0
$$

thus:

$$
\left(\frac{p^{2}}{\omega_{n}^{* 2}}-1\right)=\sum_{i=1}^{N} \frac{L_{i}^{\theta} \Gamma_{\theta, i} / I_{\theta}}{\left(\frac{\omega_{i}^{2}}{\omega_{n}^{* 2}}-1\right)}=\sum_{i=1}^{N} \frac{m_{i}^{*} h^{* 2}}{\left(\frac{\omega_{i}^{2}}{\omega_{n}^{* 2}}-1\right)}
$$

It is possible to calculate $\omega_{n}^{*}$ using an iterative method or any equation solver (e.g., Matlab and Excel). The eigenvectors are calculated by placing the values of $\omega_{n}^{*}$ in Equation 26 and assuming $\phi_{0, n}^{*}$ equal to one (or any arbitrary number different than zero). Interestingly, Equation 28 is almost identical to the corresponding one derived by Kelly in [23] for base-isolated structures equipped with rubber isolation devices assumed to respond in pure shear.

For the rocking podium systems, the same procedure is applied and the modes and frequencies are calculated. The eigenvectors' components are:

$$
\phi_{i, n}^{*}=\phi_{0, n}^{*} \frac{2 H \Gamma_{i} \omega_{n}^{* 2}}{\omega_{i}^{2}-\omega_{n}^{* 2}} \quad \text { for } \mathrm{i}=1 \text { until } N \text { and } \mathrm{n}=0 \text { until } N
$$

naming $p_{p}^{2}=-M_{\theta} g H / I_{\theta, p}$ the rocking podium frequency, it follows that:

$$
\left(\frac{p_{p}^{2}}{\omega_{n}^{* 2}}-1\right)=\sum_{i=1}^{N} \frac{4 H^{2} \Gamma_{i} L_{i}^{h} / I_{\theta, p}}{\left(\frac{\omega_{i}^{2}}{\omega_{n}^{* 2}}-1\right)}=\sum_{i=1}^{N} \frac{m_{i}^{*}(2 H)^{2} / I_{\theta, p}}{\left(\frac{\omega_{i}^{2}}{\omega_{n}^{* 2}}-1\right)}
$$




\section{Influence of uplift on higher modes}

For soil-structure interaction, Veletsos [24] suggested that foundation flexibility only affected expressively the frequency of the first mode of structures. Similarly, for rocking foundations, Psycharis [22] suggested the same. Then, using this property, Yim and Chopra [9] demonstrated that it is possible to use a 2DOF surrogate (Equation 12] with only one mode), the response of elastic SDOF systems (using the fixed-base MDOF system's higher-modes frequencies) and a modal combination rule (SRSS) to obtain accurate results for the base shear of a MDOF system on a rocking foundation, when compared to the one obtained by the exact solution of Equation 3

Using the equations derived in Section 2.5, an analysis of how the uplifted frequencies are affected by structural parameters is performed herein. Two extreme types of building are analyzed: the shear building, which has an infinite beam to column stiffness ratio, $\rho$ in Chapter 19 of Chopra [19], and the flexural building, whose $\rho$ is zero, both with uniform story mass and stiffness. Starting with the rocking foundation case (Section 2.1), by analyzing Equation 28 it is possible to conclude that the ratio between uplifted and fixed frequency $\left(\omega_{i}^{*} / \omega_{i}\right)$ depends on the type of the building and two dimensionless quantities: the aspect ratio $\left(h_{1}^{*} / B\right)$ and the rocking to flexure frequency ratio $\left(p / \omega_{1}\right)$. This is a consequence of the modal properties $\left(\Phi\right.$ and $\left.\omega_{1} / \omega_{i}\right)$ being constant for a building type and independent of the building's natural frequency. The results obtained for a 10 stories shear and flexural building are presented in Figure 3. the aspect ratio varied from 2.5 to 20 and the first mode period from 0.4 to 4 seconds, consequently $p / \omega_{1}$ varied from 0.05 to 0.4 . It is noticeable that the frequency ratios are much higher for the flexural building than for the shear building (both $\omega_{1}^{*} / \omega_{1}$ and $\omega_{2}^{*} / \omega_{2}$ ). And, contrary to what was observed by previous researchers, the uplifted second mode frequency can be $60 \%$ higher than the fixed base one for a 10 stories shear building, and it can get more than doubled for the flexural building. Moreover, the frequency ratios are almost identical for typical values of $p / \omega_{1}$ and, for very slender buildings, the frequency ratios reach a plateau. Lastly, the same analysis was run for 5 and 15 stories buildings, and the aforementioned conclusions are valid for them.
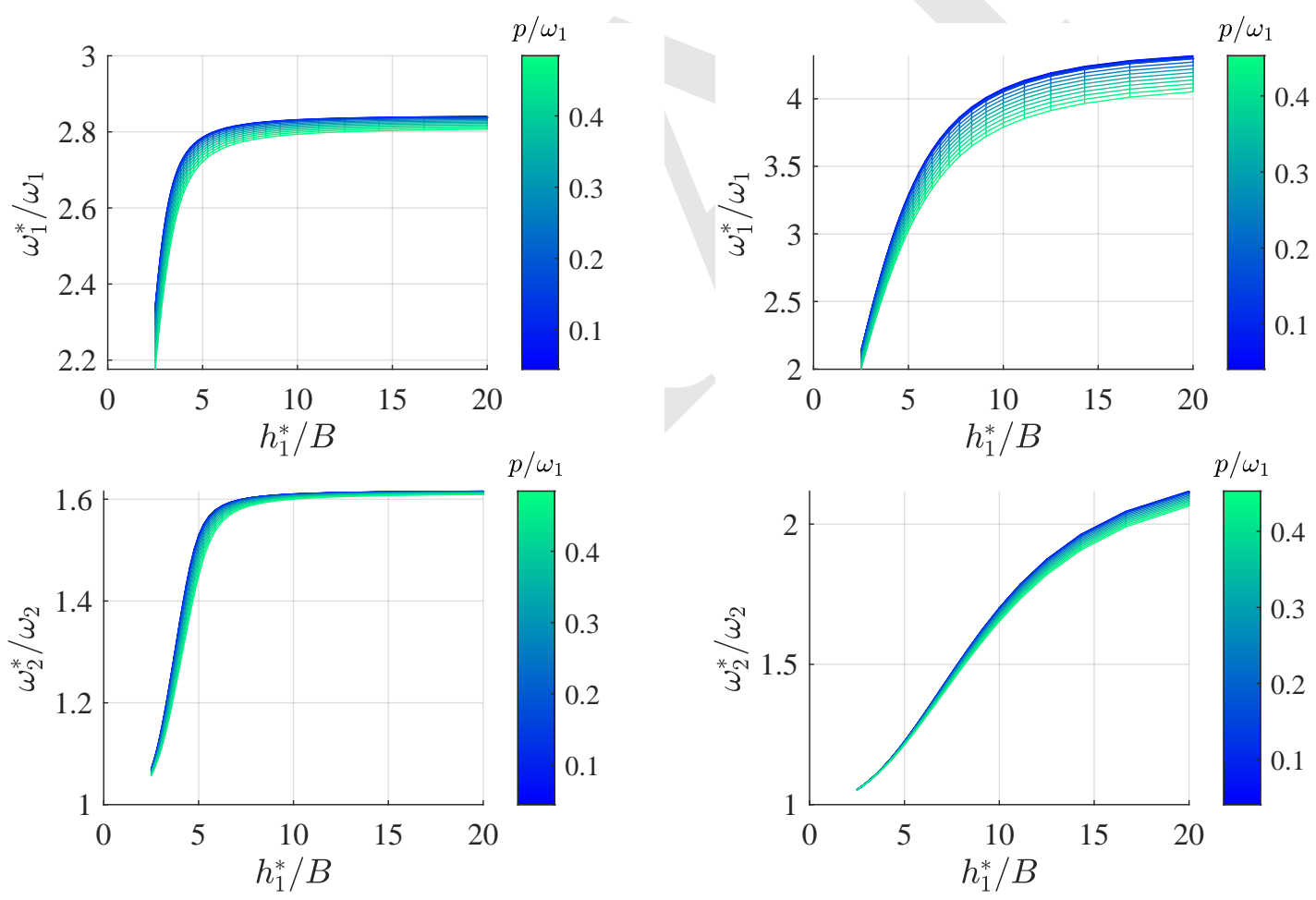

Figure 3: Uplifted to fixed frequency ratios ( $\omega_{1}^{*} / \omega_{1}$ on top and $\omega_{2}^{*} / \omega_{2}$ on bottom) for a 10 stories shear building (left) and a 10 stories flexural building (right) on a rocking foundation.

Due to the similarity of Equations 28 and 30 , it is expected that the same conclusions will be valid for rocking podium systems. Analyzing Equation 30 and the ratio $4 H^{2} \Gamma_{i} L_{i}^{h} / I_{\theta, p}$, it is evident that the ratio between uplifted and fixed frequency $\left(\omega_{i}^{*} / \omega_{i}\right)$ depends on the type of the building (due to constant modal properties) and four dimensionless quantities: the column aspect ratio $(H / B)$, the rocking to flexure frequency ratio $\left(p / \omega_{1}\right)$ and the rocking podium mass ratios $\left(\gamma=m_{b} /\left(N_{c} m_{c}\right)\right.$ and $\left.\eta=\iota^{t} \boldsymbol{m}_{\boldsymbol{s}} \boldsymbol{\iota} / m_{b}\right)$. 
In Figure 4, the frequency ratios calculated for a 10 stories shear and flexural building are presented. For simplicity, it is assumed that the cap beam has the same mass as a building floor $(\eta=10)$, and the mass ratio between the cap beam mass and column masses $(\gamma)$ is fixed equal 10. The aspect ratio was varied between 3 and 15 and the first mode period from 0.4 to 4 seconds, consequently $p / \omega_{1}$ varied from 0.12 to 1.2 . In contrast to the rocking foundation, the rocking podium's parameter $p / \omega_{1}$ notably affects the frequency ratio, it is more important than $H / B$ for the first mode frequency ratio $\left(\omega_{1}^{*} / \omega_{1}\right)$. Moreover, for the rocking podium, the second mode uplifted frequency is not expressively different from the fixed base one, it is around $10 \%$ to $30 \%$ larger. Lastly, the same analysis is ran for $\gamma=1$ and $\gamma=20$, for $\eta=5$ and $\eta=20$, and for 5 and 15 stories and the aforementioned conclusions are the same.
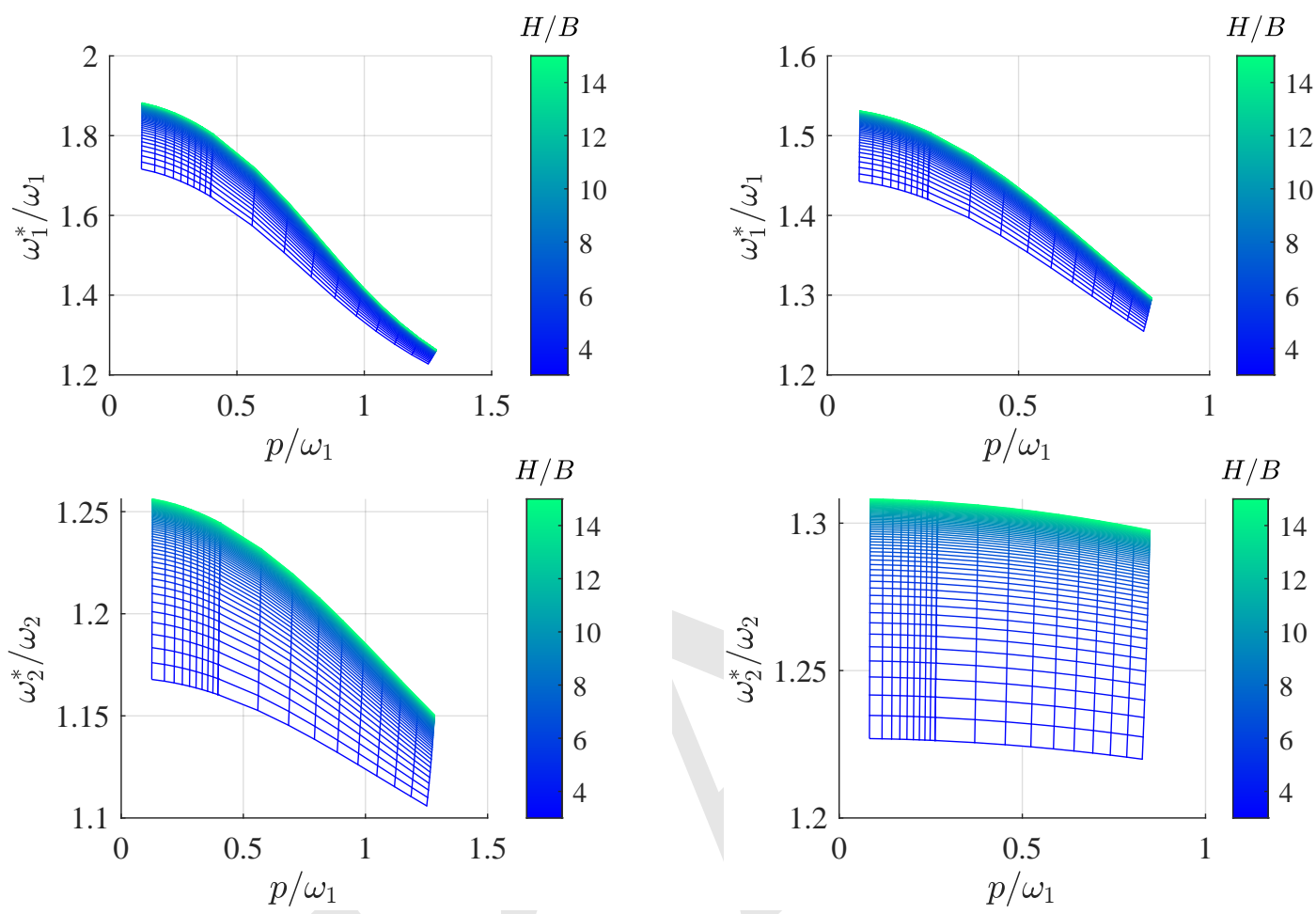

Figure 4: Uplifted to fixed frequency ratios ( $\omega_{1}^{*} / \omega_{1}$ on top and $\omega_{2}^{*} / \omega_{2}$ on bottom) for a 10 stories shear building (left) and a 10 stories flexural building (right) on a rocking podium $(\gamma=10$ and $\eta=10)$.

\section{Influence of higher modes on the total response:}

Yim and Chopra [9] suggested a simplified analysis procedure for a MDOF system on a rocking foundation. It combines the response of a reduced model (Equation 12 considering only the first mode) and the response of higher modes computed by standard procedures (SDOF system subjected to a ground motion $\Gamma_{n} \ddot{u}_{g}$, with frequency $\omega_{n}$ and damping ratio $\zeta$ ) that do not account the interaction with foundation uplift. The procedure proposed by Yim and Chopra [9] provides accurate estimates of the MDOF system response because foundation uplift is driven by base overturning moment, which is known to be dominated by the first mode and slightly affected by higher modes. Additionally, they observed that the response of the higher modes coordinates $\left(q_{n}\right.$, in which $\left.n \geq 2\right)$ are similar to the response of the SDOF computed by standard procedures (Figure 5 and Figure 6 on the left).

Meanwhile, for the rocking podium system, the uplift of the columns is driven by the base shear of the building times the height of the column $(2 H)$. Contrary to the base moment, the base shear is known to be influenced by higher modes effects, as it is proportional to $m_{n}^{*}$ times the spectral acceleration (often higher for high frequencies). However, the 2DOF surrogate gives accurate results for the podium system too (Figure 5 and 6 on the right). The explanation lies in the fact that the first mode dominates the total overturning moment on the block caused by the elastic motion of the building, whereas the block overturning moment caused by higher modes often have opposites signs and the same order of magnitude, that are lower than the first mode one, thus canceling out.

A ten story shear building $(\rho=\infty)$ is analyzed for 2 different earthquake records and for rocking foundation and rocking podium cases. The building characteristics are very similar, the one in a rocking foundation has: $T_{1}=1 \mathrm{~s}$, 

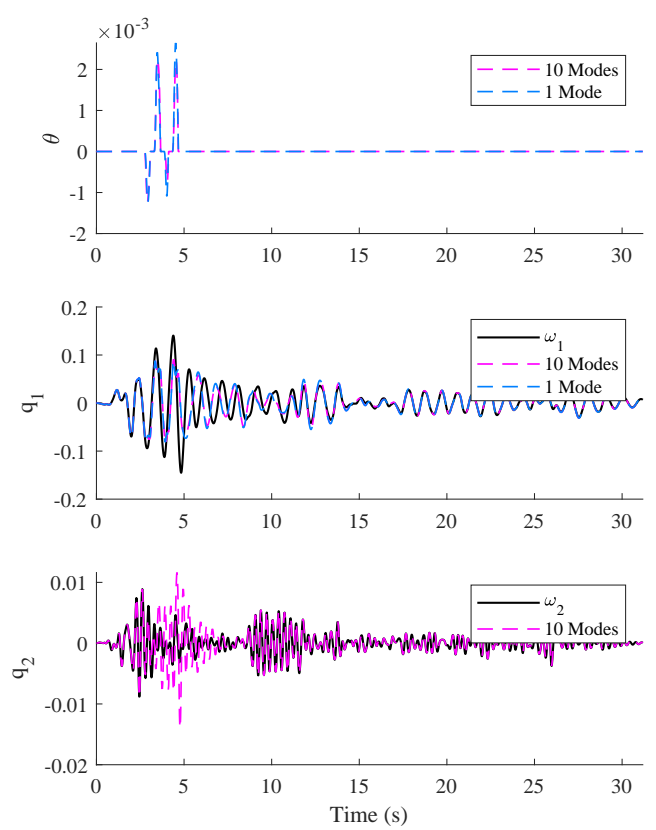
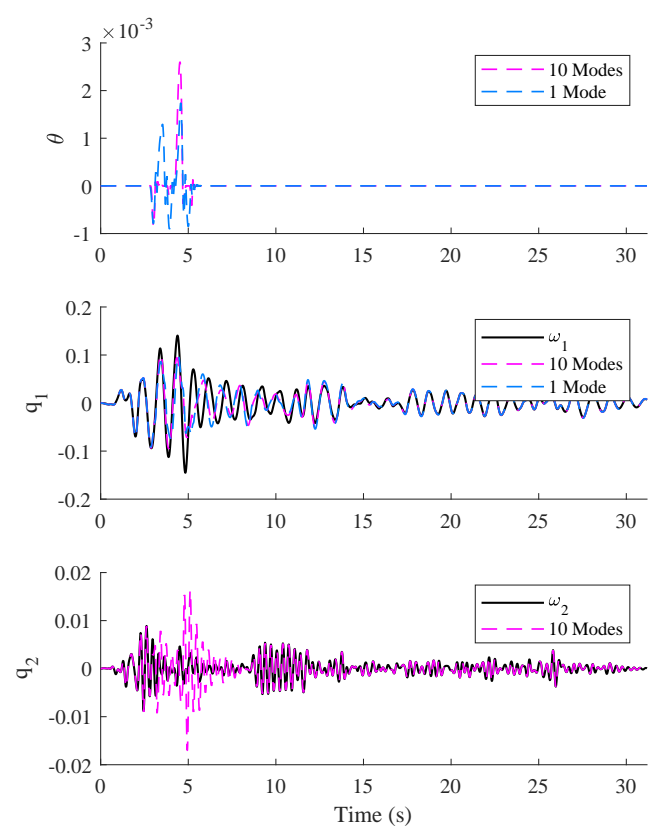

Figure 5: Ten story shear building on a rocking foundation (left) and on a rocking podium (right) with natural period of $1 \mathrm{~s}$ and aspect ratio of 5, subjected to El Centro ground motion.

$p^{2}=-0.45 s^{-2}, h^{*} / B=5, m_{t} g B / I_{\theta}=0.1 s^{-2}$, and the one on a rocking podium: $T_{1}=1 s, p^{2}=-0.47 s^{-2}$, $H / B=5, M_{\theta} g B / I_{\theta, p}=0.09 s^{-2}$. Both systems were analyzed fully (accounting all ten modes) and in a surrogate version (accounting only one mode). The first and second mode responses $\left(q_{1}\right.$ and $\left.q_{2}\right)$ are compared to the SDOF system's response calculated for $\Gamma_{n} \ddot{u}_{g}$, frequency $\omega_{n}$ and damping ratio $\zeta$ in Figures 5 and 6 .

It is evident in Figures 5 and 6 that the 2DOF surrogate predicts well the rocking rotation angle $(\theta)$ for all four cases. Also, the response of the first mode $\left(q_{1}\right)$ differs significantly from the SDOF system's response $\left(\omega_{1}\right)$ when $\theta$ is large, whereas for a small $\theta$ it approaches $\omega_{1}$. The second mode response $\left(q_{2}\right)$ is similar to the one of the respective SDOF system $\left(\omega_{2}\right)$, as found by Yim and Chopra [9]. However, $q_{2}$ has a spiky behavior during the rocking motion compared to $\omega_{2}$.

The results in Figures 5 and 6 (right side) are counter to the conclusions of Bantilas et al [15], as they observed that all vibration modes are excited by the impact of the rocking columns and considered advisable to use MDOF models for reducing the uncertainty in the response of rocking podium systems. To understand how the higher vibration modes affect the structural base shear, and consequently the overturning moment in the columns, the total base shear $\left(V_{b, t o t a l}\right)$ and the base shear due to each mode response $\left(V_{b, n}\right.$ due to $\left.q_{n}\right)$ are calculated separately. But, instead of plotting them separately, the sum of them is plotted to highlight how adding a new mode adds accuracy to the prediction. The result is presented in Figure 7 and it is possible to conclude that the rocking behavior will be dominated by the first mode base shear and that the addition of higher modes does not add much accuracy. Moreover, the total base shear of the MDOF system is bounded by the critical base shear, which is the resisting moment divided by $2 H\left(V_{c}=M_{\theta} g B /(2 H)\right.$ ). Yet, this is not observed in the rocking foundation system, in which the base shear oscillates around the first-mode critical one $\left(V_{c, 1}^{*}=m_{t} g B / h_{1}^{*}\right)$, as presented in Figure 8 .

Therefore, based on these preliminary results, the surrogate model that accounts for only the first mode of vibration (2DOF) is accurate enough for estimating the displacements of system on a rocking foundation and on a rocking podium. Yet, for estimating the base shear, for the rocking podium system, only the surrogate response is enough as it is a conservative estimate. While for the rocking foundation system, the sum of the second mode response is advisable, either by adding the time history responses of the 2DOF surrogate and the SDOF system calculated by standard procedures [9], or by a modal combination rule (e.g., square root sum of squares) of the maximum base shear of these two. 

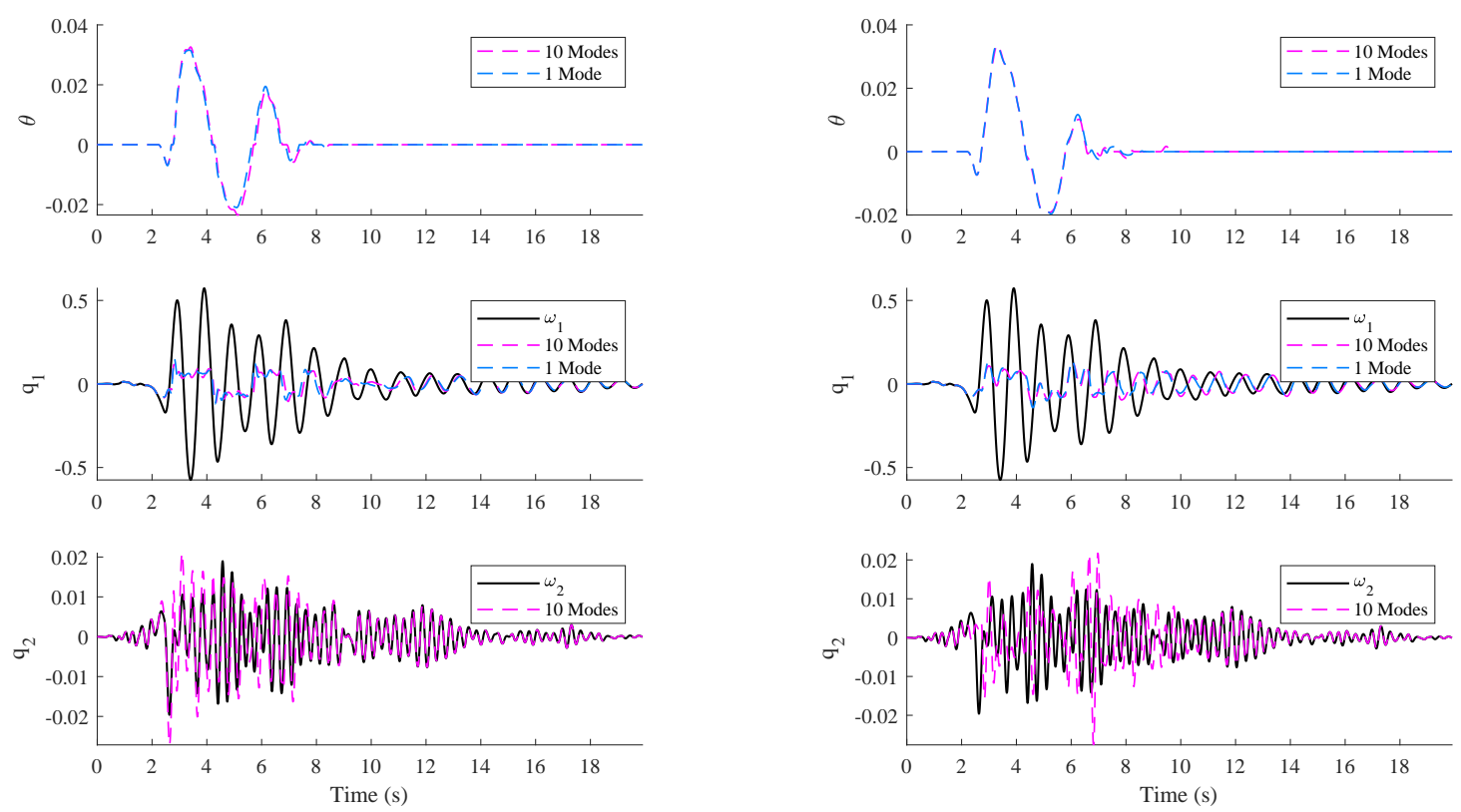

Figure 6: Ten story shear building on a rocking foundation (left) and on a rocking podium (right) with natural period of $1 \mathrm{~s}$ and aspect ratio of 5 , subjected to Northridge ground motion.
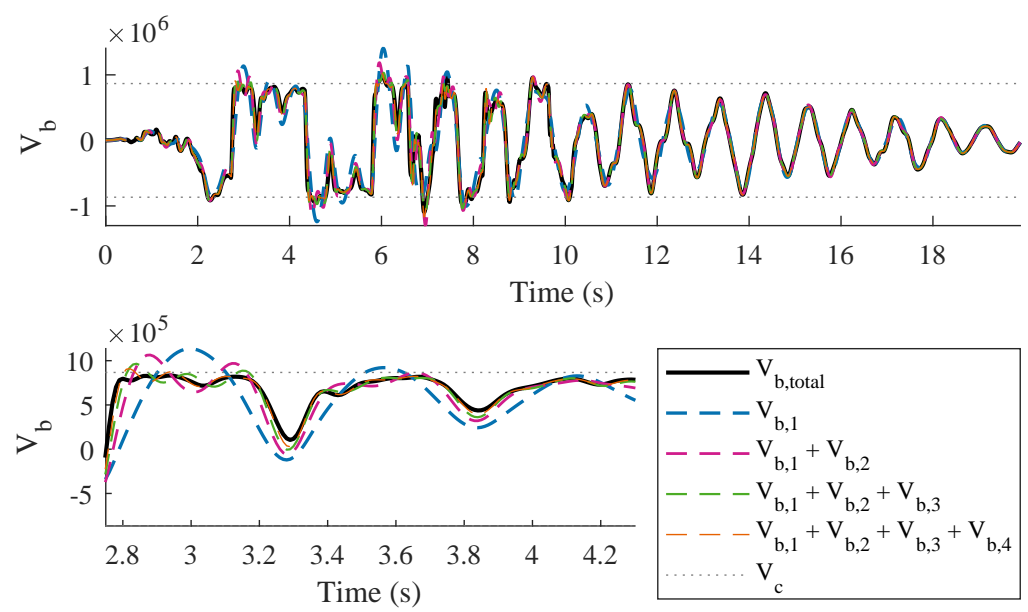

Figure 7: Total base shear $\left(V_{b, \text { total }}\right)$ and base shear caused by each mode response $\left(V_{b, n}\right)$ for the rocking podium system of Figures 5 and 6 subjected to Northridge earthquake record.

\section{2DOF surrogate accuracy}

In the previous section, it is shown that the $2 \mathrm{DOF}$ surrogate of the 10 stories shear building predicts well the displacement and rotation response of the MDOF system. The $2 \mathrm{DOF}$ also predicts fairly well the base shear, that is known for being affected by higher modes responses. This is an important feature for the rocking podium system, as it governs the initiation of overturning of the columns. Yet, the results presented previously are specific to a single building (10 story shear building, $T_{1}=1 \mathrm{~s}$ ). In this section, the accuracy of the $2 \mathrm{DOF}$ surrogate, for both rocking foundation and rocking podium systems, is investigated for different buildings, of varying vibration period, number of stories and aspect ratio. 

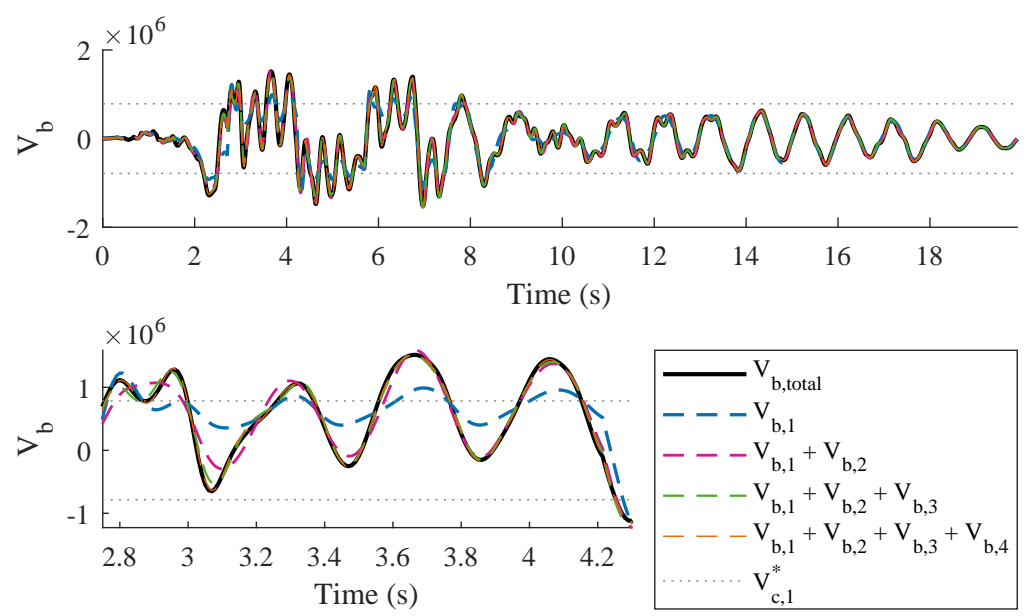

Figure 8: Total base shear $\left(V_{b, t o t a l}\right)$ and base shear caused by each mode response $\left(V_{b, n}\right)$ for the rocking foundation system of Figures 5 and 6 subjected to Northridge earthquake record.

A hundred random shear buildings are generated: they have a random number of stories (uniformly distributed between 5 and 15), random aspect ratio (uniformly distributed between 5 and 10), and random natural period (between 0.4 and 2 seconds). The full model is run for the Northridge earthquake record (not scaled for the rocking foundation case, and scaled by 0.2 for the rocking podium as it overturns in most of the cases otherwise). Then, the analysis is also run for the respective 2DOF surrogate. The results of both analysis are plotted in Figure 9 for the foundation case and in Figure 10 for the podium case. There is a clear agreement between the results: the coefficient of determination $\left(R^{2}\right)$ for the maximum elastic displacement $\left(u_{\max }\right)$ is 0.95 for the rocking foundation and 0.86 for the rocking podium. And the coefficient of determination of the maximum rocking angle $\left(\theta_{\max }\right)$ is 0.999 for the rocking foundation and 0.82 for the rocking podium.

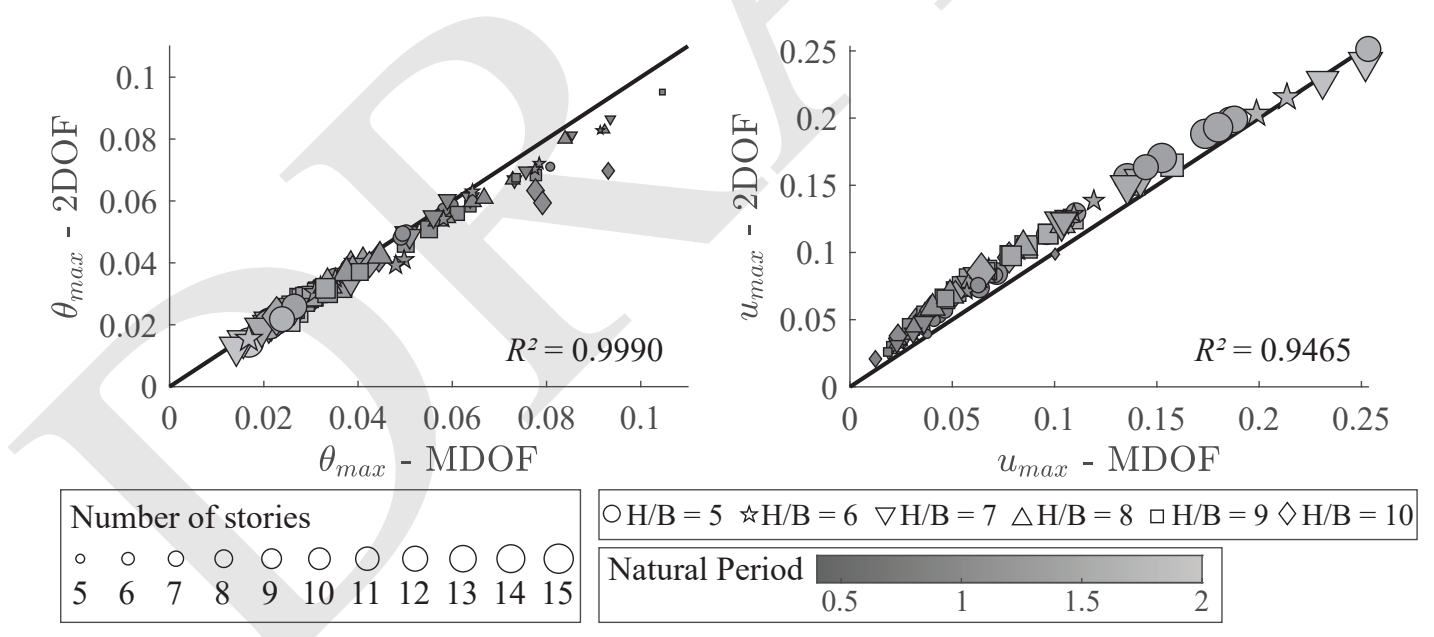

Figure 9: Structural response of hundred random buildings on a rocking foundation with different number of stories, aspect ratio and first mode period (MDOF) and the response of the respective 2DOF surrogate for the Northridge earthquake record.

\section{Conclusions}

To use rocking motion as base-isolation in seismic design, the interaction between rocking and flexure must be understood well and surrogate models should be available for the construction of earthquake spectra. In this paper, the equations of motion of a MDOF system on a rocking foundation and on a rocking podium in modal coordinates version are presented, and component mode synthesis is applied to produce surrogate models. Using these equations, analytical 

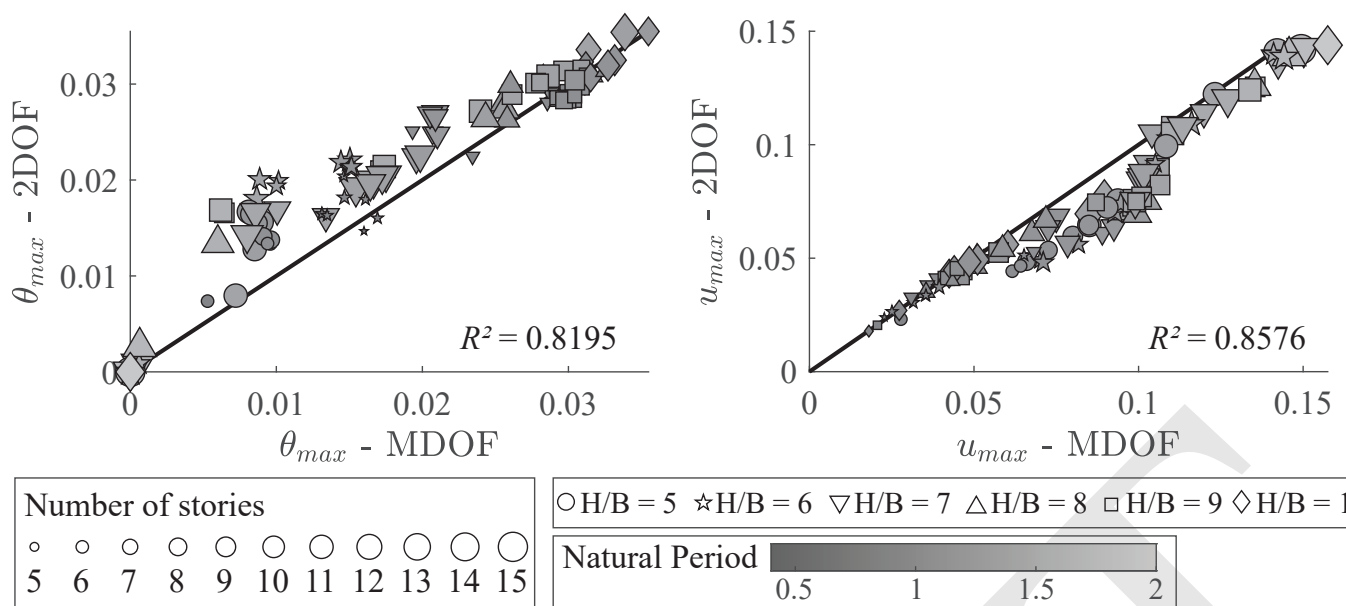

$\mathrm{OH} / \mathrm{B}=5$ « $\mathrm{H} / \mathrm{B}=6 \nabla \mathrm{H} / \mathrm{B}=7 \triangle \mathrm{H} / \mathrm{B}=8 \square \mathrm{H} / \mathrm{B}=9 \diamond \mathrm{H} / \mathrm{B}=10$

Natural Period 0.5 1

Figure 10: Structural response of a hundred random buildings on a rocking podium with different number of stories, aspect ratio and first mode period (MDOF) and the response of the respective 2DOF surrogate for the Northridge earthquake record scaled to 0.2 times.

expressions to calculate the vibration properties of the uplifted system are derived and used to define which parameters affect the most those properties. It is found that, for the rocking foundation case, the first mode uplifted frequency can be four times higher than the fixed-base one, and the second mode uplifted frequency can be almost double of the fixed-base one. For the rocking podium case, the first and second mode fixed-base frequencies are less affected by uplift, it is 10 to $60 \%$ higher for the first mode and 10 to $30 \%$ for the second. Moreover, by analyzing the building responses in modal coordinates, for both rocking podium and rocking foundation, the first mode response dominates the overturning moment, therefore a surrogate model with only the first modal coordinate (a 2DOF surrogate) is considered accurate enough to represent the system. Lastly, a hundred different shear buildings were generated, by varying periods, number of stories and aspect ratios, and their displacement and rotation dynamic response of the MDOF system and the 2DOF surrogate were compared. It is shown that, for the hundred random buildings analyzed, the surrogate is very accurate as the coefficient of determination between the responses is greater than 0.81 for maximum rocking angle and 0.85 for maximum roof displacement, for both rocking podium and rocking foundation cases.

\section{Acknowledgments}

This research is supported by the Swiss National Science Foundation (SNSF) under Grant 200021_184805 and the Swiss Federal Institute of Technology (ETH) Zurich. The authors gratefully acknowledge this funding. The methods, results, opinions, findings, and conclusions presented in this report are those of the authors and do not necessarily reflect the views of the funding agencies.

\section{Financial disclosure}

None reported.

\section{Conflict of interest}

The authors declare no potential conflict of interests.

\section{References}

[1] G. W. Housner, "The behavior of inverted pendulum structures during earthquakes," Bulletin of the seismological society of America, vol. 53, no. 2, pp. 403-417, 1963.

[2] N. Makris and M. F. Vassiliou, "Planar rocking response and stability analysis of an array of free-standing columns capped with a freely supported rigid beam," Earthquake Engineering \& Structural Dynamics, vol. 42, no. 3, pp. 431-449, 2013. 
[3] Y. Cherepinskiy, "Seismic isolation of buildings with application of the kinematics bases," in 13th World Conference on Earthquake Engineering, 2004.

[4] J. Beck and R. Skinner, "The seismic response of a reinforced concrete bridge pier designed to step," Earthquake Engineering \& Structural Dynamics, vol. 2, no. 4, pp. 343-358, 1973.

[5] K. McManus, "The seismic response of bridge structures free to rock on their foundations," ME Thesis at University of Canterbury, 1980.

[6] R. Sharpe and R. Skinner, "The seismic design of an industrial chimney with rocking base," Bulletin of the New Zealand Society for Earthquake Engineering, vol. 16, no. 2, pp. 98-106, 1983.

[7] J. W. Meek, "Effects of foundation tipping on dynamic response," Journal of the Structural Division, vol. 101, no. 7, pp. 1297-1311, 1975.

[8] I. N. Psycharis, "Dynamic behavior of rocking structures allowed to uplift," 1981.

[9] S. C.-S. Yim and A. K. Chopra, "Simplified earthquake analysis of multistory structures with foundation uplift," Journal of Structural Engineering, vol. 111, no. 12, pp. 2708-2731, 1985.

[10] A. K. Chopra and S. C.-S. Yim, "Simplified earthquake analysis of structures with foundation uplift," Journal of Structural Engineering, vol. 111, no. 4, pp. 906-930, 1985.

[11] G. Oliveto, I. Calio, and A. Greco, "Large displacement behaviour of a structural model with foundation uplift under impulsive and earthquake excitations," Earthquake engineering \& structural dynamics, vol. 32, no. 3, pp. 369-393, 2003.

[12] M. F. Vassiliou, R. Truniger, and B. Stojadinović, "An analytical model of a deformable cantilever structure rocking on a rigid surface: development and verification," Earthquake engineering \& structural dynamics, vol. 44, no. 15 , pp. 2775-2794, 2015.

[13] S. Acikgoz and M. J. DeJong, "Analytical modelling of multi-mass flexible rocking structures," Earthquake engineering \& structural dynamics, vol. 45, no. 13, pp. 2103-2122, 2016.

[14] J. Bachmann, M. F. Vassiliou, and B. Stojadinović, "Dynamics of rocking podium structures," Earthquake Engineering \& Structural Dynamics, vol. 46, no. 14, pp. 2499-2517, 2017.

[15] K. E. Bantilas, I. E. Kavvadias, and L. K. Vasiliadis, "Seismic response of elastic multidegree of freedom oscillators placed on the top of rocking storey," Earthquake Engineering \& Structural Dynamics, vol. 50, no. 5, pp. 1315-1333, 2021.

[16] K. E. Bantilas, I. E. Kavvadias, L. K. Vasiliadis, and A. Elenas, "Seismic fragility and intensity measure investigation for rocking podium structures under synthetic pulse-like excitations," Earthquake Engineering \& Structural Dynamics, 2021.

[17] W. C. Hurty, "Dynamic analysis of structural systems using component modes," AIAA journal, vol. 3, no. 4, pp. 678-685, 1965.

[18] R. R. Craig Jr and M. C. Bampton, “Coupling of substructures for dynamic analyses.," AIAA journal, vol. 6, no. 7, pp. 1313-1319, 1968.

[19] A. Chopra, Dynamics of Structures: Theory and Applications to Earthquake Engineering. Always learning, Pearson, 2017.

[20] S. Acikgoz and M. J. DeJong, "The interaction of elasticity and rocking in flexible structures allowed to uplift," Earthquake Engineering \& Structural Dynamics, vol. 41, no. 15, pp. 2177-2194, 2012.

[21] R. Truniger, M. F. Vassiliou, and B. Stojadinović, "An analytical model of a deformable cantilever structure rocking on a rigid surface: experimental validation," Earthquake engineering \& structural dynamics, vol. 44, no. 15 , pp. 2795-2815, 2015.

[22] I. N. Psycharis, "Dynamic behavior of rocking structures allowed to uplift," Tech. Rep. EERL 81-02, California Institute of Technology, 1982.

[23] J. M. Kelly, Earthquake-resistant design with rubber. Springer, 1993.

[24] A. S. Veletsos, "Dynamics of structure-foundation systems," Structural and geotechnical mechanics, pp. 333-361, 1977. 